\title{
Distribution of Intersegmental Inputs to Nonspiking Local Interneurons and Motor Neurons in the Locust
}

\author{
Gilles Laurent and Malcolm Burrows \\ Department of Zoology, University of Cambridge, Cambridge CB2 3EJ, England
}

\begin{abstract}
Intersegmental interneurons in a mesothoracic population that receive inputs from extero- and proprioceptors on a middle leg of a locust (Laurent, 1987a) make direct synaptic connections with nonspiking local interneurons and motor neurons controlling the movements of the ipsilateral hindleg. Of 25 direct connections that were established, $80 \%$ are excitatory, and $60 \%$ are made with nonspiking interneurons. Two or more intersegmental interneurons with outputs of either polarity and with different or overlapping receptive fields can converge onto one metathoracic nonspiking interneuron.
\end{abstract}

Direct connections were found with motor neurons of the coxotrochanteral, femorotibial, and tibiotarsal joints of the hindleg. An intersegmental interneuron can make divergent connections with several motor neurons of the same pool or with a motor neuron and a nonspiking interneuron presynaptic to it. By virtue of the lateral inhibitory connections between nonspiking interneurons controlling different pools of motor neurons (Burrows, 1979), an intersegmental interneuron can excite directly one pool and disynaptically inhibit the antagonistic pool.

Metathoracic nonspiking interneurons also receive inputs from afferents of mechanoreceptors on a hindleg. They thus have 2 receptive fields: one on the hindleg whose motor neurons they control and one on the ipsilateral middle leg, provided by inputs from the mesothoracic intersegmental interneurons. Nonspiking local interneurons are thus a point of convergence of local and intersegmental pathways. The effectiveness of the intersegmental pathways can thus be modified by local inputs to the nonspiking interneurons.

Coordination of the limbs during terrestrial locomotion depends on a balance between central commands and sensory feedback from the environment (for reviews, Grillner, 1981; Bässler, 1983; Graham, 1985; Hasan and Stuart, 1988). During walking, a limb gencrates sensory feedback from its own surface and joint mechanoreceptors, so that its movements can be stabilized and corrected when subjected to unexpected perturbations. These corrective, local reflexes must not, however, jeopardize the overall stability of the animal and must, therefore, be under both central control and peripheral influences from the other limbs.

\footnotetext{
Received Oct. 28, 1988; revised Jan. 30, 1989; accepted Feb. 20, 1989.

This work was supported by grant NS16058 from NIH and by a grant from the SERC, IJK to M.B. and by a Hasseblad Foundation grant to G.J. G.L. is a Locke Research Fellow of the Royal Society. We thank our Cambridge colleagues for their help and advice during the course of this work.

Correspondence should be addressed to G. Laurent, Department of Zoology, University of Cambridge, Downing Street, Cambridge CB2 3EJ, England.

Copyright (C) 1989 Society for Neuroscience $0270-6474 / 89 / 093019-11 \$ 02.00 / 0$
}

Central modulation of local reflexes occurs in humans (Capaday and Stein, 1986; Dietz et al., 1986), higher mammals (Forssberg, 1979), lower vertebrates (Sillar and Roberts, 1988), and invertebrates (DiCaprio and Clarac, 1980; Sillar and Skorupski, 1986). An afferent signal can, for example, evoke either a "resistance" reflex with negative feedback or an "assistance" reflex with positive feedback, depending on the phase of the step cycle in which it occurs. The few known mechanisms responsible for these phase-dependent changes and "reflex reversals" are of central origin, since they can be expressed in deafferented preparations.

By contrast, modulation of local reflexes of one leg by sensory inputs to another region of the body is poorly documented because, during walking, it is not possible to study peripheral influences in the absence of central ones. In the locust, the pathways responsible for local reflexes of the legs are becoming well understood. Mechanosensory afferents make direct excitatory connections with both motor neurons and nonspiking local interneurons (Burrows, 1987a; Burrows et al., 1988; Laurent and Burrows, 1988a; Laurent and Hustert, 1988) and disynaptic inhibitory connections with these neurons, through a layer of spiking local interneurons (Burrows and Siegler, 1982; Siegler and Burrows, 1983; Burrows, 1987b). These connections underlie resistance and avoidance reflexes observed reliably in restrained animals (Siegler and Burrows, 1986; Burrows, 1987a). We do not know, however, if and how these local reflexes can be affected or controlled by peripheral inputs to other parts of the body, such as the adjacent legs.

During active movements, such as walking, the reflex responses of one leg will be dependent on the action of the other legs. Sensory information about the movements and position of one leg is generally conveyed to the circuits controlling an adjacent ipsilateral leg by populations of intersegmental interneurons (Laurent, 1987a, b, 1988; Laurent and Burrows, 1988a). We describe here the output connections of one population of these intersegmental interneurons, which provide information about the state of the ipsilateral middle leg to the local circuits controlling a hindleg. We show that the mesothoracic intersegmental interneurons connect in parallel with metathoracic motor neurons and with nonspiking interneurons presynaptic to them and could, therefore, influence the expression of reflexes of a hindleg.

\section{Materials and Methods}

Adult locusts Schistocerca gregaria (Forskål) of either sex were used from our laboratory culture. They were mounted ventral side up in Plasticine, with the tibia and tarsus of the left middle leg and the femur, tibia, and tarsus of the left hindleg free to move. The meso- and metathoracic ganglia were exposed and stabilized on a wax-coated platform, 
Figure 1. Excitatory connection between a mesothoracic intersegmental interneuron and a metathoracic nonspiking local interneuron. 4 , Extension of the mesothoracic tibia evokes a burst of spikes in the intersegmental interneuron and a barrage of EPSPs in the metathoracic nonspiking interneuron. The EPSPs indicated by the asterisk are not all explained by the spikes in this intersegmental interneuron. Flexion evokes IPSPs in the intersegmental interneuron, and the EPSPs in the nonspiking interneuron stop. $B$, Superimposed sweeps triggered from the spikes in the intersegmental interneuron reveal EPSPs at a constant latency in the nonspiking interneuron. $C$, The connection is confirmed by signal averaging. Each EPSP is the average of 128 occurrences of the presynaptic spike. $D$, Diagram of the pathways revealed. The triangles represent excitatory synapses. Calibration: horizontal $A, 0.5 \mathrm{sec}, B$, $6.5 \mathrm{msec}, C, 9.7 \mathrm{msec}$; vertical $A, 4 \mathrm{mV}$, $B, 1.7 \mathrm{mV}$.

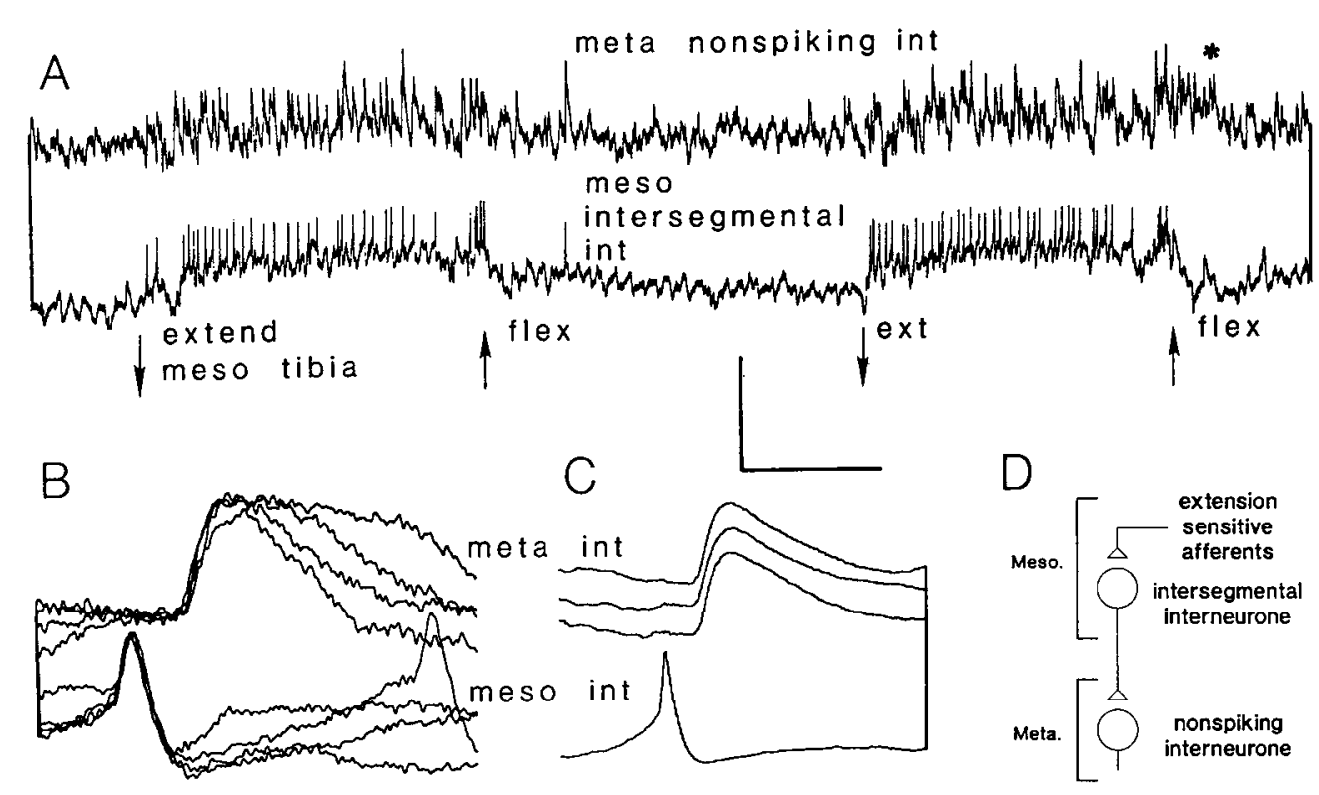

and their sheaths were treated for 1-2 min with an approximately $0.1 \%$ $(\mathrm{wt} / \mathrm{vol})$ solution of protease (Sigma type XIV) in saline. The thoracic cavity was perfused with saline throughout the experiments at a temperature of $20-22^{\circ} \mathrm{C}$.

Simultaneous intracellular recordings were made in the mesothoracic ganglion from an intersegmental interneuron in a known population (Laurent, 1986, 1987a, b) and in the metathoracic ganglion from an ipsilateral nonspiking local interneuron (Burrows and Siegler, 1978; Siegler and Burrows, 1979) or a hindleg motor neuron. Recordings, using glass micropipettes filled with $2 \mathrm{M}$ potassium acetate, were made from the somata of mesothoracic interneurons, from the neuropilar processes of metathoracic nonspiking interneurons, and from either the somata or neuropilar processes of metathoracic motor neurons. All the mesothoracic intersegmental interneurons that were studied have the following morphological characteristics and belong to the same population (Laurent, 1987a): somata in a cluster lateral at the outer edge of a posterior connective of the mesothoracic ganglion, extensive branches in neuropil ipsilateral to the soma, an axon in the ipsilateral posterior connective, and a smaller array of branches in the ipsilateral metathoracic neuromere.

The receptive fields of the mesothoracic intersegmental interneurons and of the metathoracic nonspiking interneurons were determined by mechanical stimulation of receptors on the legs using procedures similar to those previously described (Siegler and Burrows, 1986; Laurent, 1987b). The motor effects of a nonspiking interneuron (Burrows, 1980) were determined by injecting depolarizing current through the microelectrode and observing the evoked movements, or the pattern of motor spikes recorded from a muscle or its nerve. The motor neurons were identified by the intracellular injection of current and observation of the evoked movements of a hindleg.

All recordings were stored on a 7-channel Racal FM tape recorder for later averaging and display on either a Gould ES 1000 printer or a digital oscilloscope and $X-Y$ plotter. Signal averaging was performed with a digital oscilloscope or digitized traces from the oscilloscope and a microcomputer. The results are from experiments in 81 animals.

\section{Results}

\section{Connections with metathoracic neurons}

A mesothoracic intersegmental interneuron makes direct connections with specific nonspiking local interneurons in the ipsilateral half of the metathoracic ganglion. The mesothoracic interneuron in Figure 1 is excited by extension and inhibited by flexion of the tibia of the middle leg ipsilateral to its soma, neuropilar branches, and axon. Full details of the response prop- erties of this intersegmental interneuron are given in Laurent (1986, figures 1, 2). The spikes evoked in this interneuron by extension are accompanied by a barrage of EPSPs in a metathoracic nonspiking local interneuron recorded simultaneously (Fig. 1A). During flexion, IPSPs replace the spikes in the intersegmental interneuron, and the EPSPs in the metathoracic nonspiking interneuron no longer occur. Each spike in the intersegmental interneuron is followed consistently after a constant delay of $2.4 \mathrm{msec}$ by an EPSP in the metathoracic nonspiking interneuron (Fig. $1 B$ ). The EPSPs are seen clearly in signal averages (Fig. 1C), and the pathways revealed are shown diagrammatically in Figure $1 D$.

The EPSPs evoked by intersegmental interneurons in this and other nonspiking interneurons were always recorded from neuropilar branches where they have half-width durations of 5-15 msec, rise times measured from $10-90 \%$ of peak amplitude of about $1 \mathrm{msec}$, and amplitudes up to $4 \mathrm{mV}$. No homosynaptic depression or facilitation was found when the EPSPs were evoked by current injected into a single intersegmental interneuron, but heterosynaptic facilitation was sometimes suspected when EPSPs in one nonspiking interneuron were elicited by several, converging intersegmental interneurons excited by the same mesothoracic sensory stimulus.

Intersegmental interneurons in this mesothoracic population also make direct connections with specific ipsilateral hindleg motor neurons. For example, the intersegmental interneuron in Figure 2 inhibits a levator of the metathoracic trochanter (Fig. $2 A$ ). Spikes in the interneuron evoked by pulses of depolarizing current are followed by a barrage of IPSPs and a consequent decrease in the frequency of spikes in the motor neuron (Fig. $2 A$ ). The inhibition of the levator motor neuron produced in this way can be sufficient to result in a slow depression of the hind femur. Each spike in the intersegmental interneuron is followed consistently by an IPSP in the motor neuron after a constant latency of $2 \mathrm{msec}$ (Fig. $2 B$ ). The pathway revealed is shown in Figure $2 C$.

To provide a better approximation of the central latency to the PSPs in the metathoracic neurons, the conduction velocity 


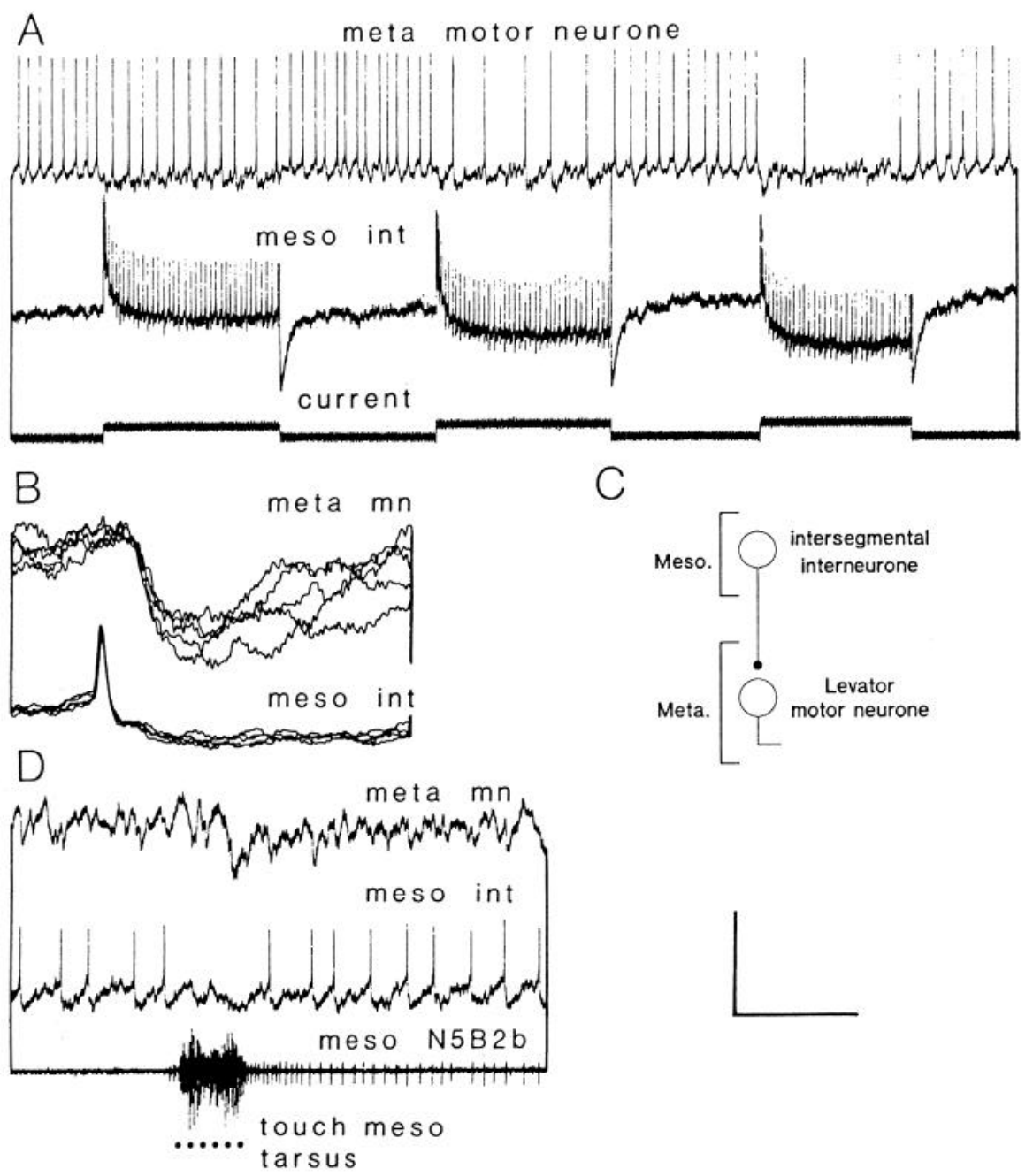

Figure 2. Inhibitory connection between a mesothoracic intersegmental interneuron and a metathoracic levator trochanteris motor neuron. $A$, Spikes evoked in the intersegmental interneuron by injection of current cause IPSPs in the motor neuron and reduce the frequency of its spikes. $B$, Each spike in the intersegmental interneuron is followed after a constant latency by an IPSP in the motor neuron. $C$, Diagram of the pathways revealed in $A$ and $B$. The filled circle represents an inhibitory synapse. $D$, Touching the mesothoracic tarsus evokes a burst of afferent spikes (recorded in the mesothoracic tibial nerve $5 \mathrm{~B} 2 \mathrm{~b}$ ) and an inhibition of both the mesothoracic interneuron and the metathoracic motor neuron. Calibration: horizontal $A, 1 \mathrm{sec}, B, 16 \mathrm{msec}$, $D, 0.5 \mathrm{sec}$; vertical motor neuron $A, 10$ $\mathrm{mV}, B, 1.25 \mathrm{mV}, D, 4 \mathrm{mV}$, interneuron $A, 4 \mathrm{mV}, B, 1.5 \mathrm{mV}, D, 4 \mathrm{mV} ; A, 25$ nA.

of the spikes of the intersegmental interneurons was measured. Most interneurons in this population project no farther than the metathoracic neuromere, so that there is insufficient distance to provide an accurate measure of conduction velocity. A few, however, have an axon in an abdominal connective of the metathoracic ganglion. Their axons in the meso-metathoracic connective are of the same diameter as those of interneurons that project only to the metathoracic neuromere (see Laurent, 1987a). These interneurons were, therefore, selected and simultaneous recordings were made intracellularly from their soma and extracellularly from their axon in the abdominal connective. Signal averaging was necessary to reveal the extracellular spikes. The average conduction velocity of their spikes is $1.5 \mathrm{~m} \cdot \mathrm{sec}^{-1}$, so that the central latency to a PSP in a metathoracic neuron, excluding conduction in the presynaptic axon, is between 0.7 and $1.4 \mathrm{msec}$. These results thus suggest that the connections made by the intersegmental interneurons with metathoracic nonspiking interneurons and with motor neurons are direct and chemically mediated.

This population of intersegmental interneurons consists of at least 30 interneurons (Laurent, 1987a), and the patterns of output connections made by the individuals we have sampled are summarized in Table 1. From a total of 25 established connections with metathoracic neurons, $60 \%$ (15) are made with nonspiking local interneurons and $36 \%$ (9) with motor neurons. One postsynaptic spiking neuron could not be identified, but no effects on midline spiking local interneurons (Burrows and Siegler, 1984 ) were ever detected. Eighty percent ( 20 of 25 ) of all the established connections are excitatory. Excitatory synapses with nonspiking interneurons are, therefore, the most frequently encountered type of connection (44\%). Connections with motor neurons controlling the coxotrochanteral, femorotibial and tib-

Table 1. Details of the 25 connections found between mesothoracic intersegmental interneurons and metathoracic nonspiking interneurons and motor neurons

\begin{tabular}{|c|c|c|c|c|c|c|c|}
\hline $\begin{array}{l}\text { Output } \\
\text { neuron }\end{array}$ & Non- & Mo & $r$ p & s & & & \\
\hline $\begin{array}{l}\text { Sign of } \\
\text { connection } \\
(\%)\end{array}$ & $\begin{array}{l}\text { local } \\
\text { ints. } \\
(60 \%)\end{array}$ & $\begin{array}{l}\mathrm{D} . \\
\mathrm{Tr}\end{array}$ & $\begin{array}{l}\mathrm{L} . \\
\mathrm{Tr}\end{array}$ & $\begin{array}{l}\text { E. } \\
\text { Ti }\end{array}$ & $\begin{array}{l}\text { Fl. } \\
\text { Ti }\end{array}$ & $\begin{array}{l}\mathrm{R} . \\
\mathrm{U} .\end{array}$ & $\begin{array}{l}\text { Uniden } \\
\text { tified } \\
(4 \%)\end{array}$ \\
\hline
\end{tabular}

\section{Excitatory}

$\begin{array}{llllllll}(80) & 11 & 1 & 1 & 1 & 2 & 3 & 1\end{array}$

Inhibitory

The numbers give the total connections of a particular type that have been found. D.Tr, L.Tr, depressor and levator trochanteris; E.Ti, Fl.Ti, extensor and flexor tibiae; R.U., retractor unguis. 

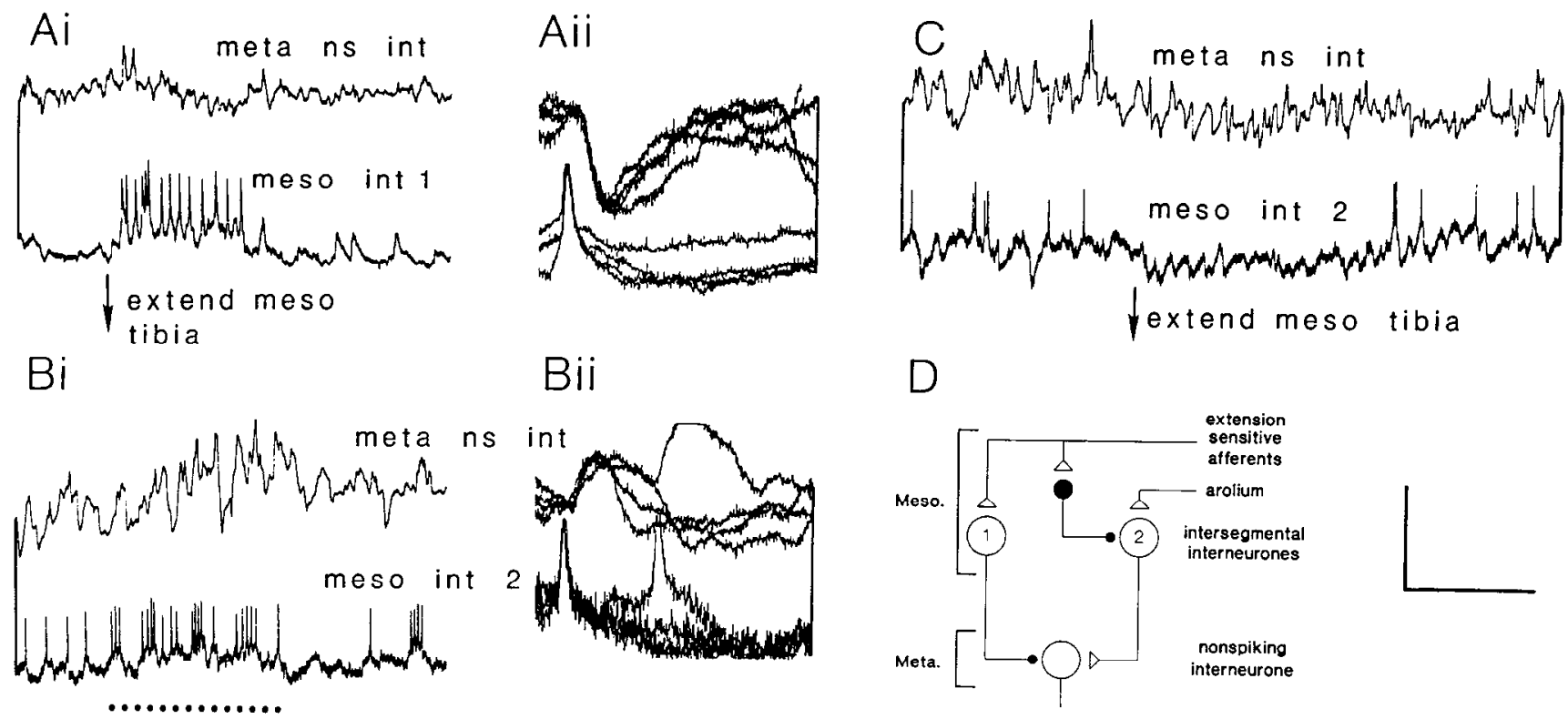

$\mathrm{Bii}$
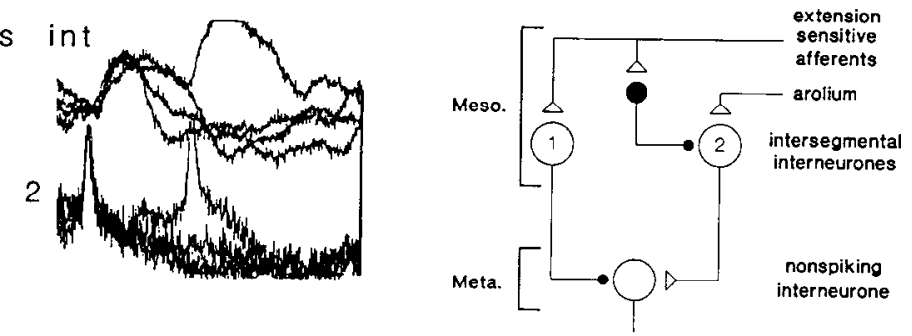

touch meso arolium

Figure 3. Convergence of 2 mesothoracic interneurons onto a single metathoracic nonspiking interneuron. $A i$, Extension of the mesothoracic tibia evokes a burst of spikes in a mesothoracic interneuron (int 1 ) and IPSPs in a metathoracic nonspiking interneuron (upper trace). Aii, The spikes in the intersegmental interneuron cause consistent IPSPs in the nonspiking interneuron. Bi, Touching the mesothoracic arolium evokes a burst of spikes in a second mesothoracic interneuron (int 2) and a large depolarization of the metathoracic nonspiking interneuron. Bii, The spikes in this intersegmental interneuron cause consistent EPSPs in the nonspiking interneuron. $C$, Extending the mesothoracic tibia inhibits int 2 , thereby preventing it from exciting the metathoracic nonspiking local interneuron. $D$, The simplest pathways that explain the observed connections. $A$ mesothoracic spiking local interneuron (filled soma) inhibits intersegmental interneuron, 2. Calibration: horizontal $A i, B i, C, 0.5$ sec, $A i i, B i i, 24$ $\mathrm{msec}$; vertical, intersegmental interneurone $A i, B i, C, 10 \mathrm{mV}, A i i, B i i, 8 \mathrm{mV}$, nonspiking interneuron $A i, B i, C, 25 \mathrm{mV}, A i i, B i i, 8 \mathrm{mV}$.

iotarsal joints of a hindleg have all been found, suggesting that the mesothoracic interneurons have a widespread influence on the pools of motor neurons controlling the hindleg.

\section{Convergence onto metathoracic neurons}

Convergence of several mesothoracic intersegmental interneurons onto a metathoracic nonspiking interncuron or motor neuron was indicated wherever a direct connection from an intersegmental interneuron was found. In Figure 1A, not all the EPSPs elicited in the nonspiking interneuron by extension of the mesothoracic tibia can be explained by the spikes in the mesothoracic interneuron that is recorded simultaneously (asterisk, Fig. 1A). Similarly, in Figure 2D, the inhibition of the metathoracic trochanteral levator motor neuron when the mesothoracic tarsus is brushed cannot be caused by the mesothoracic interneuron recorded simultaneously as its spikes are inhibited by this stimulus. Several mesothoracic intersegmental interneurons must, therefore, converge onto particular metathoracic neurons.

In Figure 3, a metathoracic nonspiking interneuron is inhibited by extending the tibia of the ipsilateral middle leg (Fig. 3Ai) and excited by touching exteroceptors on the ipsilateral mesothoracic arolium at the distal end of the tarsus (Fig. 3Bi). These effects, which define 2 regions of its mesothoracic receptive field, are due to converging inputs from at least 2 mesothoracic intersegmental interneurons. The first interneuron is excited by tibial extension (Fig. 3Ai) and causes 1:1 IPSPs in the nonspiking interneuron (Fig. 3Aii). The second interneuron, recorded sequentially in the same locust, is excited by touching the mesothoracic arolium (Fig. 3Bi) and causes 1:1 EPSPs in the nonspiking interneuron (Fig. 3Bii). Furthermore, the second intersegmental interneuron is inhibited by extension of the mesothoracic tibia, so that during this movement, the inhibition of the nonspiking interneuron due to inputs from the first intersegmental interneuron (Fig. 3Ai) is supplemented by a cessation of excitation from the second (Fig. 3C). The pathways revealed are shown in Figure $3 D$.

\section{Divergence to several metathoracic neurons}

A single mesothoracic intersegmental interneuron can connect with several metathoracic neurons. The interneuron in Figure 4 , for example, is excited by tactile stimulation of the mesothoracic tarsus and makes excitatory connections with 2 metathoracic retractor unguis motor neurons (Fig. 4, $A, B$ ). A spike in the interneuron is reliably followed after a constant latency by an EPSP in one of the motor neurons (Fig. 4C). Signal averaging shows that these EPSPs occur with the same latency in both motor neurons (Fig. 4D). The role played by these connections is demonstrated by altering the membrane potential of the mesothoracic interneuron. At its normal resting potential, the interneuron spikes a few times during each mesothoracic stimulus, and this leads to one or 2 spikes in a postsynaptic retractor unguis motor neuron (Fig. $4 A$ ). If the mesothoracic interneuron is hyperpolarized so that the same stimuli now fail to evoke spikes, the excitation of the metathoracic retractor motor neuron is reduced (Fig. $4 E$ ). Nevertheless, the motor neuron is still excited, presumably by converging inputs from other mesothoracic intersegmental interneurons. The connections underlying these responses are shown in Figure $4 F$.

Information from a single intersegmental interneuron can also diverge to a metathoracic motor neuron and a nonspiking local interneuron presynaptic to it. For example, a simultaneous recording from a metathoracic nonspiking interneuron and a post- 

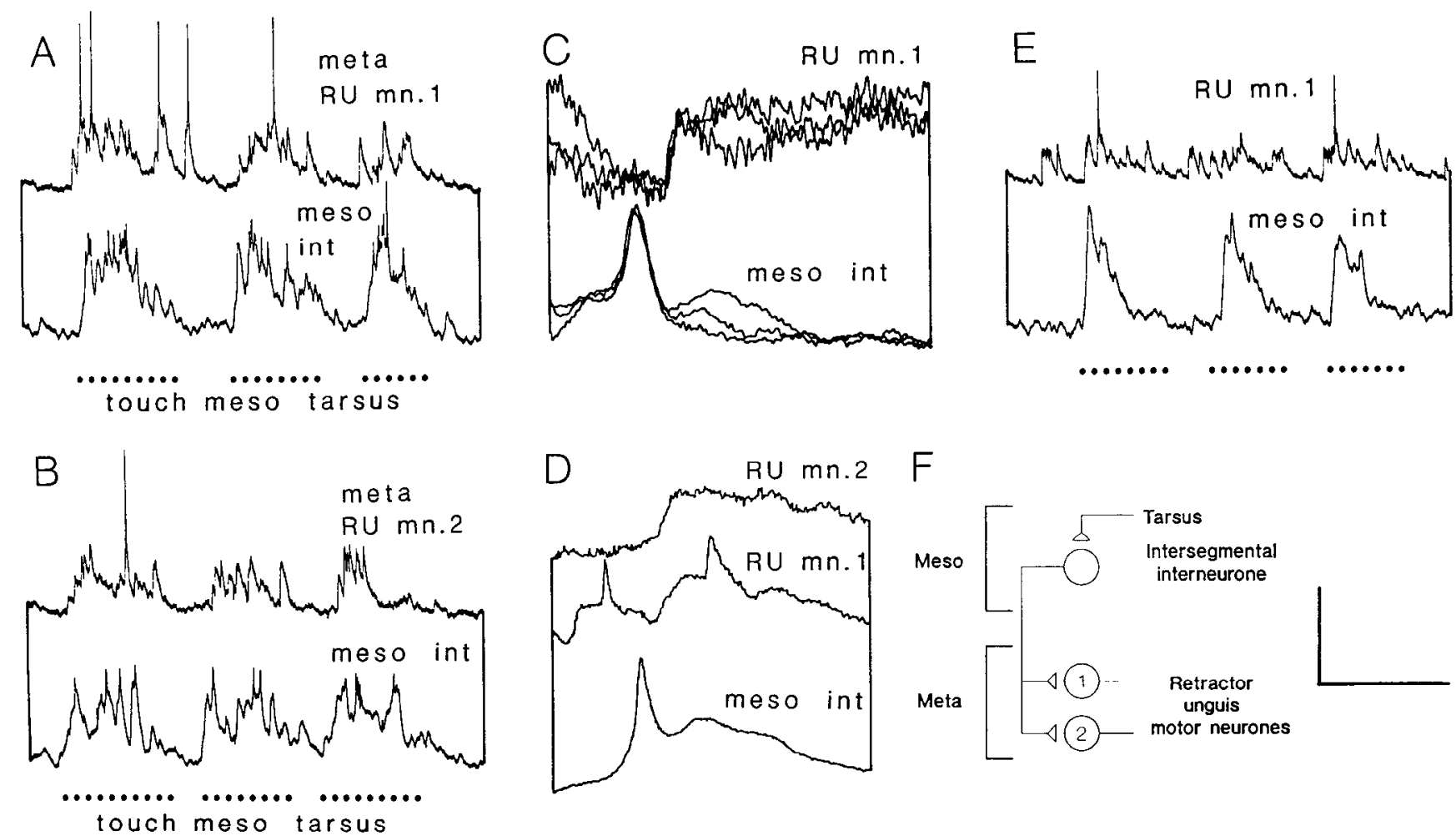

Figure 4. Divergence of a mesothoracic intersegmental interneuron to 2 metathoracic motor neurons. $A$, Touching the mesothoracic tarsus evokes a burst of spikes in the mesothoracic intersegmental interneuron and in a metathoracic retractor unguis motor neuron $(R U m n$. 1$) . B$, The same stimulus also excites a second metathoracic retractor unguis motor neuron $(R U m n .2)$, recorded later in the same experiment. $C$, Spikes in the mesothoracic interneuron are followed by consistent EPSPs in the first motor neuron. $D$, Signal averaging (16 sweeps) shows that spikes of the intersegmental interneuron evoke EPSPs with the same latency in both motor neurons recorded sequentially. $E$, If the intersegmental interneuron is held hyperpolarized while the mesothoracic tarsus is touched (dots), the excitation of an RU mn. is reduced. $F$, Diagram of the pathways revealed. Calibration: horizontal $A, B, 0.7 \mathrm{sec}, C, 6.5 \mathrm{msec}, D, 14 \mathrm{msec}, E, 0.5 \mathrm{sec}$; vertical RU mn. $1 A, 14 \mathrm{mV}, C, 6 \mathrm{mV}, E, 24 \mathrm{mV} ; \mathrm{RU} \mathrm{mn} .2 B, 14$ $\mathrm{mV}$; interneuron $A, B, E, 14 \mathrm{mV}, C, 8 \mathrm{mV}$.

synaptic motor neuron can reveal common synaptic inputs when a mesothoracic stimulus is applied. A consequence of this arrangement will be an increased excitation of the motor neuron if the output connections of the nonspiking interneuron are excitatory and, by virtue of the connections of the nonspiking interneuron with several members of a motor pool or with several pools, a wider distribution of the mesothoracic signals.

A further divergence of the mesothoracic signals occurs through the inhibitory connections that metathoracic nonspiking interneurons make with each other (Burrows, 1979). Figure 5 contrasts the effects of a direct connection with a nonspiking interneuron (Fig. $5 A$ ) and an indirect effect, probably mediated by a second, interposed nonspiking interneuron (Fig. $5 B$ ). Depolarizing the mesothoracic interneuron to evoke spikes causes a hyperpolarization of the metathoracic nonspiking interneuron (Fig. 5Ai). Each of its spikes is followed by IPSPs at a constant latency, suggesting a direct connection (Fig. 5Aii, iii). Depolarizing another mesothoracic interneuron also causes a hyperpolarization of a different metathoracic nonspiking interneuron (Fig. $5 B i$ ), but this time no IPSPs are linked to the spikes, suggesting that no direct connection exists (Fig. 5Bii). The simplest explanation for this observation is that the mesothoracic interneuron directly excites a metathoracic nonspiking interneuron, which in turn inhibits the nonspiking interneuron from which the recording is made (Fig. 5Biii). The inhibitory interactions between nonspiking interneurons, therefore, provide a mechanism by which a mesothoracic intersegmental interneuron that directly affects one motor pool could exert the opposite effect on an antagonistic motor pool.

\section{Mesothoracic receptive fields of metathoracic nonspiking interneurons}

The connections made by the mesothoracic interneurons with metathoracic neurons should be placed in a behavioral context to allow a better understanding of their possible role. The activation of the mesothoracic interneurons will largely be determined by the pattern of afferent signals generated by receptors within their receptive fields on a middle leg. Whether a metathoracic neuron is affected by a mesothoracic stimulus will, therefore, be determined by the receptive fields of the interneurons that converge onto it. The mesothoracic receptive field of a metathoracic nonspiking interneuron was, therefore, compared with that of presynaptic mesothoracic intersegmental interneurons by selective stimulation of various areas of the middle leg (Fig. 6). Touching hairs on the dorsal tarsus of the ipsilateral mesothoracic leg evokes bursts of EPSPs in the nonspiking interneuron that are caused by several mesothoracic intersegmental interneurons, one of which is recorded simultaneously (Fig. 6, $A, B$ and represented as interneuron $I$ in Fig. $6 D$ ). Touching exteroceptors on the dorsal arolium strongly excites the nonspiking interneuron but only weakly excites the mesothoracic interneuron by decreasing the frequency of some 
A i

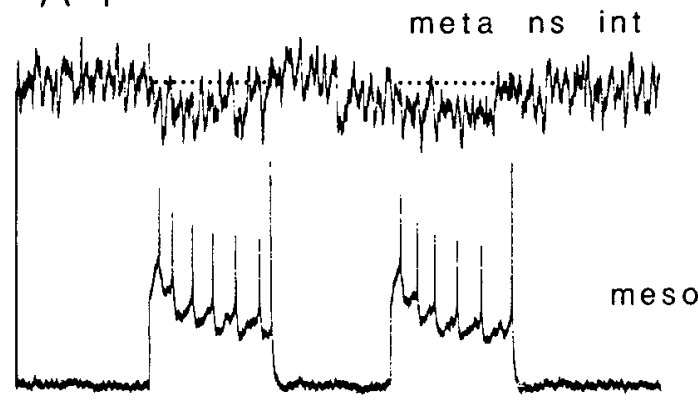

$\mathrm{B} \mathrm{i}$

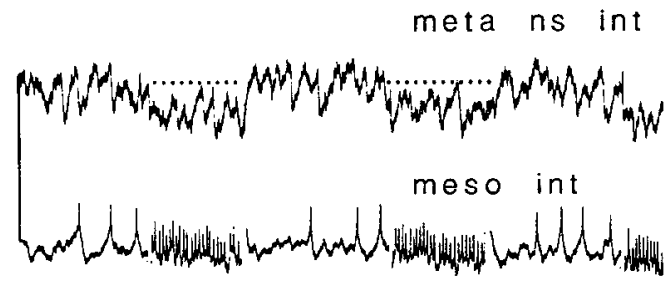

ii

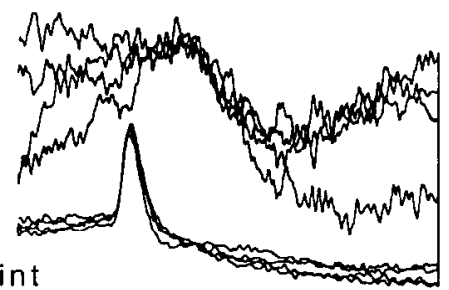

ii

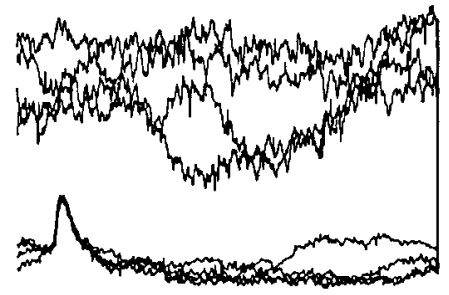

iii

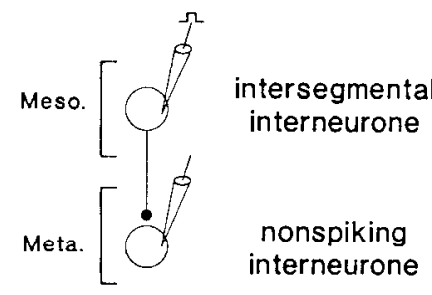

III

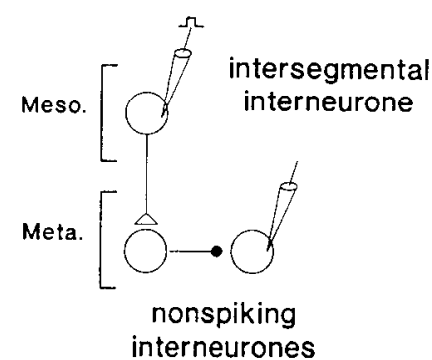

Figure 5. Direct and indirect effects of mesothoracic intersegmental interneurons on a metathoracic nonspiking interneuron. $A$, Direct effects. $A i$, Injecting depolarizing current into a mesothoracic intersegmental interneuron produces a hyperpolarization of a metathoracic nonspiking interneuron. Aii, Each spike in the intersegmental interneuron is consistently followed by an IPSP in the nonspiking interneuron, suggesting that the connection is direct. Aili, Diagram of the pathway revealed. $B$, Indirect effects. $B i$, Injecting depolarizing current into a second intersegmental interneuron to make it spike also evokes a hyperpolarization of a metathoracic nonspiking interneuron. Bii, No synaptic potential in the nonspiking interneuron is, however, linked to the spikes. Biii, The pathway probably involved. Calibration: horizontal $A i, B i, 0.5 \mathrm{sec}, A i i, 6.5 \mathrm{msec}, B i i, 14 \mathrm{msec}$; vertical, nonspiking interneuron $A i, B i, 2 \mathrm{mV}, A i i, 0.8 \mathrm{mV}, B i i, 1.7 \mathrm{mV}$, intersegmental interneuron $A i, 4 \mathrm{mV}, A i i, 3.5 \mathrm{mV}, B i, 2 \mathrm{mV}, B i i, 1.7 \mathrm{mV}$.

tonic IPSPs (Fig. 6Ci). This indicates that a second mesothoracic interneuron with an excitatory input must converge onto the nonspiking interneuron (interneuron 2 in Fig. 6D). Touching hairs on the dorsal tibia has only a weak inhibitory effect on the nonspiking interneuron but a stronger inhibitory effect on the mesothoracic intersegmental interneuron, caused by local interneurons in the mesothoracic ganglion (Fig. 6, Cii, D). Touching hairs on the ventral tibia near the spurs leads to a slight hyperpolarization of the nonspiking interneuron and an excitation of the mesothoracic interneuron (Fig. 6 Ciii). The excitation of the nonspiking interneuron by the impaled intersegmental interneuron can thus be overridden by inhibitory inputs provided in parallel by a third mesothoracic interneuron (interneuron 3 in Fig. $6 D$ ). Touching the ventral tibia proximal to the distal spurs strongly inhibits the nonspiking interneuron, possibly through the action of interneuron 3 , but has little effect on the impaled mesothoracic interneuron (Fig. 6 Civ). The mesothoracic receptive field of this metathoracic nonspiking interneuron is, therefore, the result of converging inputs from at least three types of mesothoracic intersegmental interneurons (Fig. 6D).

Twelve metathoracic nonspiking local interncurons with a receptive field on a middle leg determined by inputs from mesothoracic interneurons and a second field on the ipsilateral hindleg determined by inputs from afferents are shown in Figure 7. Most of these interneurons have receptive fields on different regions of a hindleg and middle leg, and only rarely are they the same (see interneuron 4 in Fig. 7). Most fields combine inputs from extero- and proprioceptors and can have both excitatory and inhibitory regions.

\section{Nonspiking local interneurons can alter the effectiveness of intersegmental pathways}

A consequence of the organization of the receptive fields of nonspiking interneurons into meso- and metathoracic regions is that inter- and intrasegmental effects could interact at this level. For example, during a resistance reflex of the hindleg caused by an imposed extension of the hind tibia, a metathoracic flexor tibiae motor neuron is excited, and a metathoracic nonspiking interneuron is inhibited (Fig. $8 A$ ). The nonspiking interneuron directly inhibits this flexor tibiae motor neuron (Fig. $8 B$ ). Therefore, the inhibition of the nonspiking interneuron aids the resistance reflex by decreasing its tonic inhibitory input to the flexor motor neuron. Both neurons also are affected by tactile inputs to the ipsilateral middle leg. Touching the tarsus of the middle leg evokes large EPSPs in the nonspiking interneuron and a small hyperpolarization in the motor neuron (Fig. 8Ci). By contrast, touching the tibia of the middle leg evokes EPSPs simultaneously in both neurons that probably are caused by the same mesothoracic interneuron (Fig. 8, Cii, D). If the mesoand metathoracic stimuli were to coincide, complex interactions between excitatory input from the middle leg and inhibitory input from the hindleg to the nonspiking interneuron would occur, making it difficult to predict the response of the motor neuron.

The contribution of the nonspiking interneuron to the effectiveness of the output connections of a mesothoracic intersegmental interneuron can be demonstrated in the following way. If the nonspiking interneuron is depolarized with DC current while the mesothoracic stimulus is delivered, the EPSPs evoked 

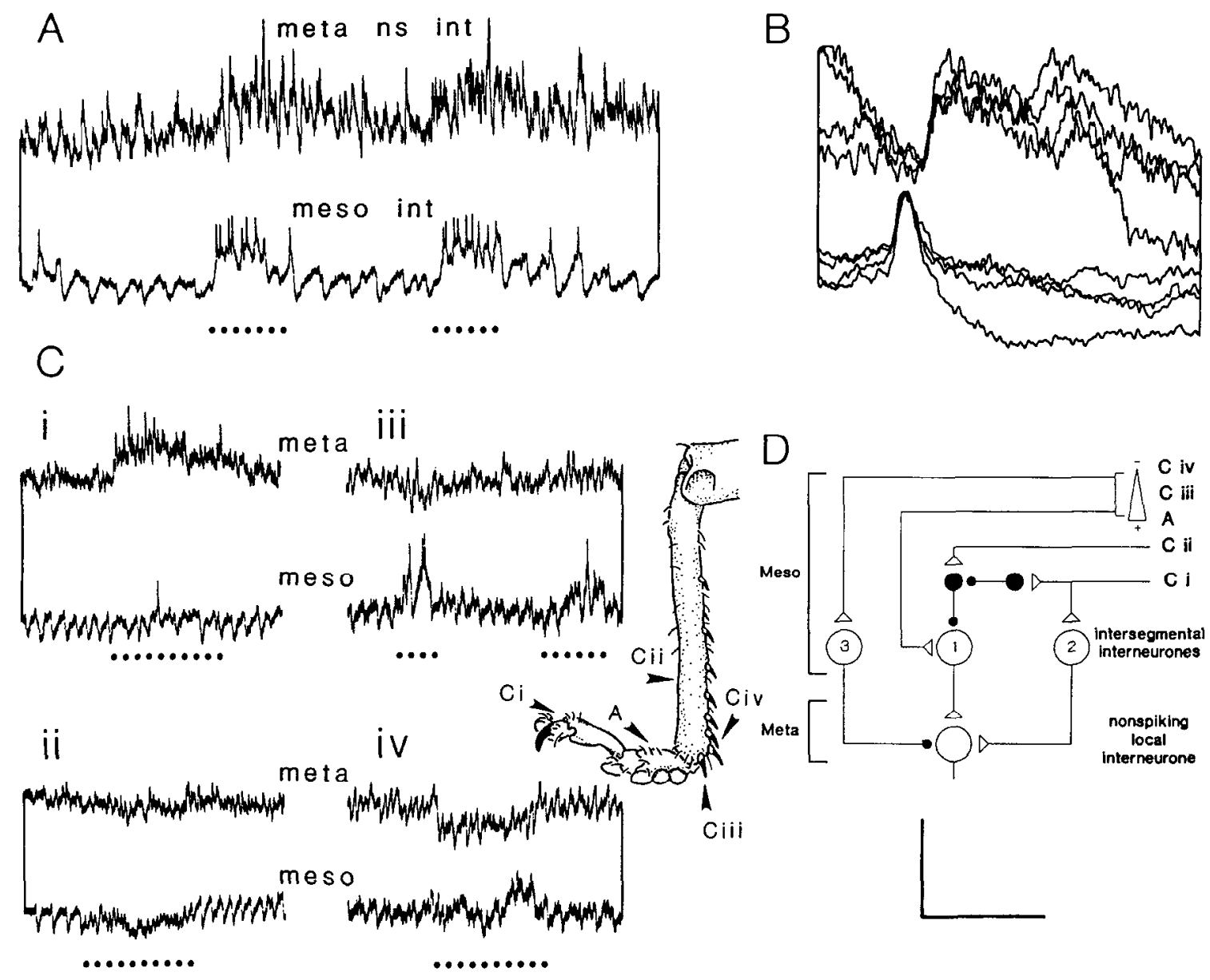

Figure 6. The derivation of the mesothoracic receptive field of a metathoracic nonspiking local interneuron. $A$, Touching hairs on the dorsal tarsus of a middle leg (dots) evokes a burst of spikes in the intersegmental interneuron and EPSPs in the nonspiking interneuron. $B$, EPSPs in the nonspiking interneuron consistently follow each spike in the intersegmental interneuron. $C$, Other regions in the mesothoracic receptive fields of the two interneurons shown on the drawing of the middle leg. $C i$, Dorsal arolium. Cii, Dorsal tibia. Ciii, Region of the spurs. Civ, Ventral tibia. $D$, Diagram of the simplest connections that can explain the observed pattern of responses. The intersegmental interneuron recorded here in A-C is labeled 1 . Interneurons 2 and 3 are additional ones required to explain the observations. The large open triangle represents a gradient of excitation and inhibition in one region of the receptive field, and the letters on this and the inset drawing of the middle leg refer to the parts of the figure showing the experimental evidence. Calibration: horizontal $A, 0.25 \mathrm{sec}, B, 6.5 \mathrm{msec}, C, 0.5 \mathrm{sec}$; vertical, intersegmental interneuron $A, C, 4 \mathrm{mV}, B, 2.5$ $\mathrm{mV}$, nonspiking interneuron $A, 2 \mathrm{mV}, B, 1.5 \mathrm{mV}, C, 4 \mathrm{mV}$.

in the flexor motor neuron are then decreased in amplitude (Fig. $8 E$ ). This can be explained by 2 concomitant factors: first, an increase in input conductance in the motor neuron due to the chemical inhibitory drive from the nonspiking interneuron, and second, an increased efficacy of the inhibitory pathway through the nonspiking interneuron. This nonspiking local interneuron is thus interposed in both intra- and intersegmental mechanosensory pathways to a flexor motor neuron. It can increase the efficacy of a metathoracic local resistance reflex but also can reduce the excitation caused in the motor neuron by the mechanical stimulation of the mesothoracic tibia. The effectiveness of both local and intersegmental pathways will thus depend on the membrane potential of the nonspiking interneuron, which will itsclf be determined by other synaptic inputs.

\section{Discussion}

Output connections of the mesothoracic interneurons

The intersegmental interneurons studied here belong to a population whose cell bodies lie at the lateral edge of a posterior connective of the mesothoracic ganglion. They make output connections with ipsilateral metathoracic nonspiking interneurons and with hindleg motor neurons. The connections appear to be direct because spikes in the intersegmental interneurons are consistently followed by PSPs at latencics of $0.7-1.4 \mathrm{mscc}$, when allowance is made for the time that the presynaptic spike takes to reach the metathoracic synaptic sites. In addition, the axonal branches of the mesothoracic interneurons (Laurent, 1987a) project to the same regions of metathoracic neuropil as the branches of the nonspiking interneurons and motor neurons (Watkins et al., 1985), indicating that anatomical synapses between the neurons are at least possible.

Most of the output connections of these intersegmental interneurons are excitatory, but some are inhibitory. We did not find any interneuron with an excitatory effect on one neuron and inhibitory effect on another. On the basis of recordings from a large number of intersegmental interneurons involved in the control of flight in the locust, Pearson and Robertson (1987) concluded that interneurons with medial cell bodies are in general inhibitory, whereas interneurons with lateral cell bodies are 

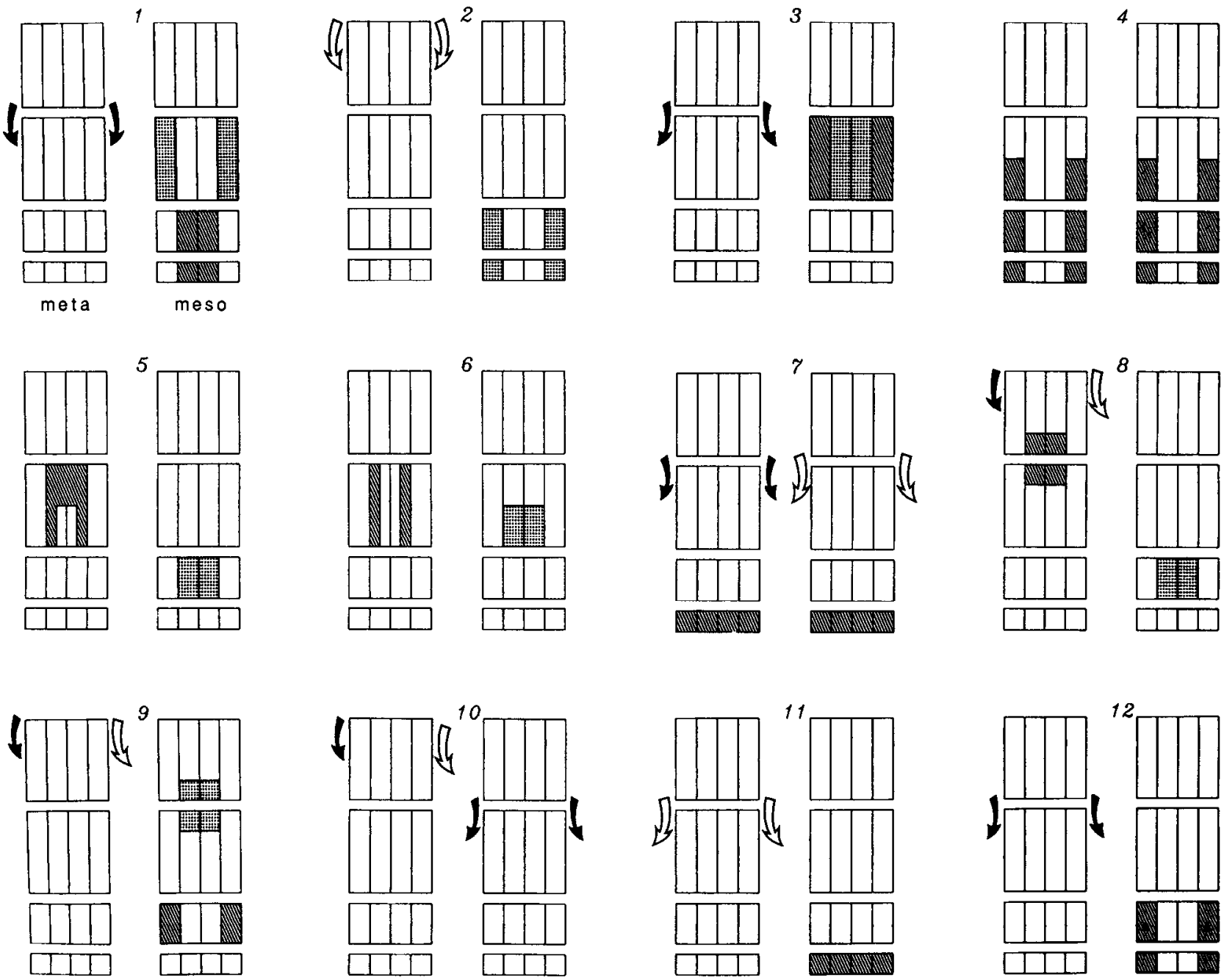

Figure 7. The mesothoracic and metathoracic receptive fields of 12 metathoracic nonspiking local interneurons. The metathoracic field is on the left of each pair, and the mesothoracic field is on the right. The surface of the leg is represented as though open by a ventral midline incision and then spread. Excitatory regions are hatched; inhibitory regions are stippled. Outward arrows indicate extension, and inward arrows indicate flexion. Filled arrows represent excitation; open arrows represent inhibition. Interneuron 4 has the same field on both the middle and hindlegs. Interneurons 1 and 12 excite the slow extensor tibiae, interneuron 7 excites a slow depressor tarsi, and interneuron 10 excites the slow depressor trochanteris motor neurons.

generally excitatory. The mesothoracic intersegmental interneurons studied here all have cell bodies in a cluster lateral to a posterior connective (Laurent, 1987a), yet some make inhibitory and others excitatory output connections. This result and the immunocytochemical evidence showing that GABA-like immunoreactivity in locust thoracic ganglia is not confined to somata close to the midline (Watson, 1986) imply that cell body position should not be used as a reliable indicator of output effects.

The intersegmental interneurons do not appear to make any conncctions with metathoracic spiking local interneurons that belong to a ventral midline population (Burrows and Siegler, 1984). These spiking local interneurons reverse the sign of afferent signals from the hindleg and, therefore, act in local afferent processing as important inhibitors of motor neurons (Burrows and Siegler, 1982), nonspiking local interneurons (Burrows, 1987b), and intersegmental interneurons (Laurent, 1987b). In many ways, they are similar to the Ia interneurons in the vertebrate spinal cord (Jankowska and Roberts, 1972), which are influenced by the central pattern-generating networks during fictive locomotion. As a result, the gain of the inhibitory pathways they mediate is strongly modulated in a phase-dependent manner (Feldman and Orlovsky, 1975). In the locust, because no connections of intersegmental interneurons have yet been found with the spiking local interneurons, it could be that the sensory signals are modified before they reach the spiking local interneurons by the presynaptic action of the intersegmental interneurons on the afferent terminals. Preliminary observations have, however, provided no evidence for such a presynaptic inhibitory mechanism.

\section{Receptive fields of nonspiking interneurons}

Intersegmental interneurons in this mesothoracic population can have receptive fields that overlap considerably (Laurent, 1987b, 1988), and their outputs may converge onto the same metathoracic target. Consider the connections of 4 intersegmental interneurons that receive inputs from 4 distinct arrays of exteroceptors on the middle leg, each represented in Figure 

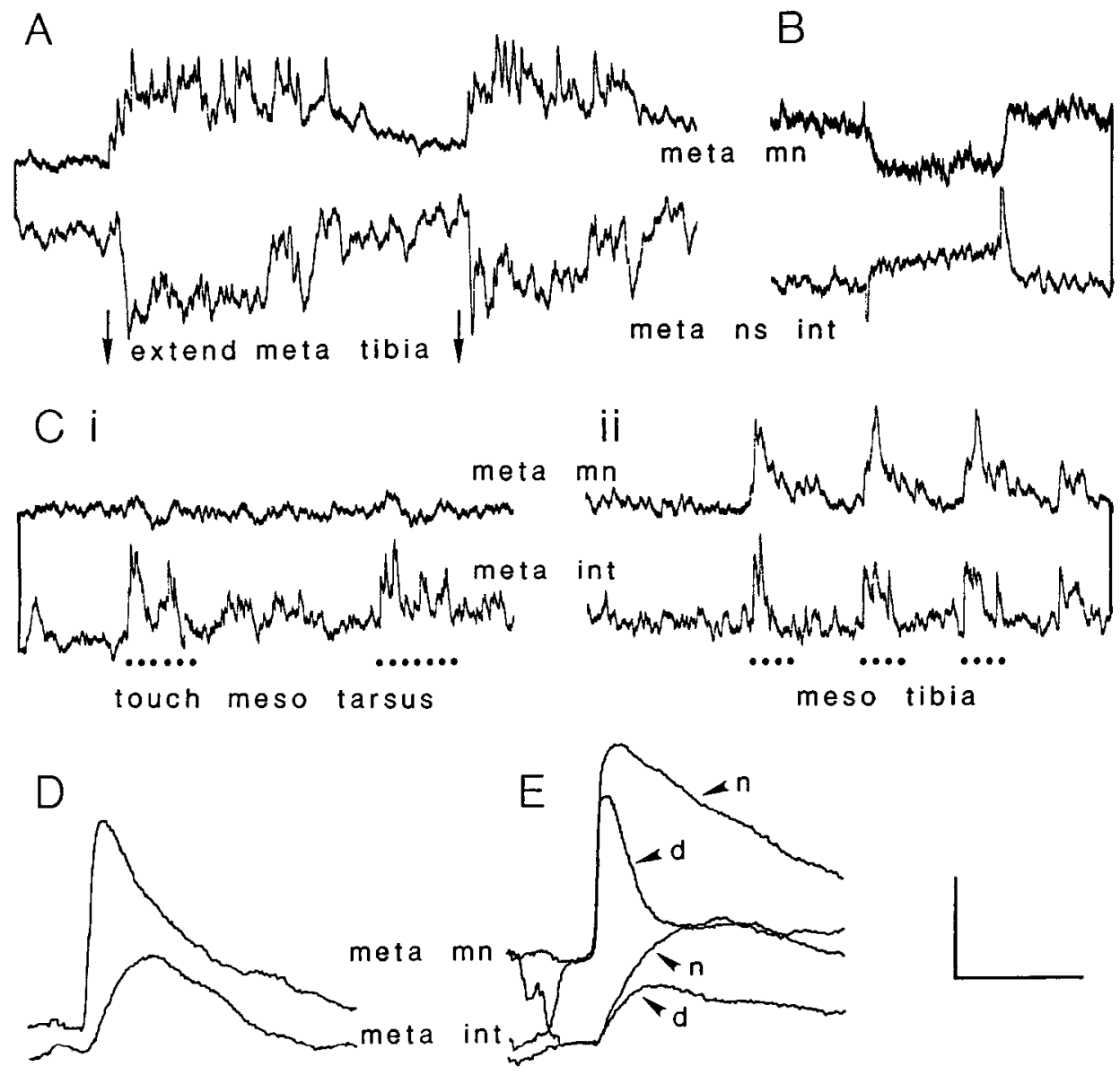

Figure 8. A metathoracic nonspiking interneuron can alter the effectiveness of an intersegmental pathway. $A$, Extending the hind tibia excites a metathoracic flexor tibiae motor neuron and inhibits a nonspiking interneuron. $B$, Depolarizing this nonspiking interneuron with a pulse of current inhibits the flexor motor neuron. $C i$, Touching exteroceptors on the mesothoracic tarsus excites the nonspiking interneuron and slightly inhibits the motor neuron. Cii, Touching the mesothoracic tibia evokes large EPSPs in both the motor neuron and the nonspiking interneuron. $D$, Signal averages of 64 sweeps triggered from the EPSPs evoked in the nonspiking interneuron by the mesothoracic tibial stimulus reveals a simultaneous EPSP in the flexor motor neuron. $E$, The normal EPSP $(n)$ evoked in the flexor motor neuron by the mesothoracic tibial stimulus is reduced in amplitude if the nonspiking interneuron is held depolarized $(d)$ during the mesothoracic tibial stimulus. Each trace is an average of 64 sweeps. Calibration: horizontal $A, B$, $C, 0.5 \mathrm{sec}, D, 19 \mathrm{msec}, E, 9.5 \mathrm{msec}$; vertical nonspiking interneuron $A, B$, $C, 8 \mathrm{mV}$, motor neuron $A, C, 8 \mathrm{mV}, B$, $4 \mathrm{mV}$.

$9 A$ by a single sensory neuron, and whose outputs converge on one metathoracic nonspiking local interneuron. Figure 9 is a diagrammatic representation incorporating the results of several experiments on different combinations of neurons.

Sensory neuron 1 excites intersegmental interneurons 1 and 2 , whose excitatory outputs then converge onto the nonspiking interneuron (Fig. 9A). Interneuron 2 has an additional excitatory receptive field provided by sensory neuron 2 that is not shared with interneuron 1 . These pathways are thus a representation of the results shown in Figure 1, where not all the EPSPs recorded in the nonspiking interneuron can be explained by spikes in one intersegmental interneuron.

Sensory neuron 1 also excites interneuron 3 , which in turn inhibits the nonspiking interneuron (Fig. 9A). The pathways of interneurons 1 and 3 represent the experimental finding in Figure $6 \mathrm{Ciii}$, where a single mechanical stimulus to one region of the middle leg simultaneously causes a slight hyperpolarization of a nonspiking interneuron and a number of EPSPs. The possible function of this surprising arrangement is considered in Figure $9 B$. Interneurons 1 and 3 have only a small part of their receptive fields that overlap. The nonspiking interneuron will have 3 main regions in its mesothoracic receptive field as a result of the convergence of inputs from these 2 mesothoracic interneurons: one that is purely excitatory because of inputs from interneuron 1 , one that is purely inhibitory because of inputs from interneuron 3 , and one that is both excitatory and inhibitory because of converging inputs from interneurons 1 and 3 . This combination appears to create a gradient of exci- tation and inhibition in its mesothoracic receptive field that blurs the boundary between excitatory and inhibitory areas. This gradient is suggested by the experimental evidence provided in Figure 6. The functional significance of such an arrangement is, however, not clear. Alternatively, the inhibitory and excitatory outputs of the 2 intersegmental interneurons could be distributed on different branches of the nonspiking interneuron so that they would act with some independence. For example, they might affect different outputs of the nonspiking interneuron and, therefore, modify different pathways (see Laurent and Burrows, 1989). A third explanation for such an arrangement could be that overlapping excitatory and inhibitory regions underlie directional selectivity in tactile movement detection.

The receptors represented by sensory neuron 1 form part of the excitatory receptive field of interneurons $1-3$ but part of the inhibitory field of interneuron 4 (Fig. 9A). The inhibition of interneuron 4 is caused by a mesothoracic spiking local interneuron (see Laurent, 1987b). Thus, the excitatory drive to the nonspiking interneuron provided by intersegmental interneurons 1 and 2 will be aided by the withdrawal of the inhibitory drive provided by interneuron 4 . These pathways represent an arrangement similar to that which could explain the experimental finding in Figure 3. There, inhibition of a metathoracic nonspiking interneuron by one intersegmental interneuron is assisted by the inhibition of a second, which would otherwise provide excitatory inputs. The inhibition of intersegmental interneurons by spiking local interneurons in the mesothoracic ganglion may thus serve two functions. First, it may sharpen 


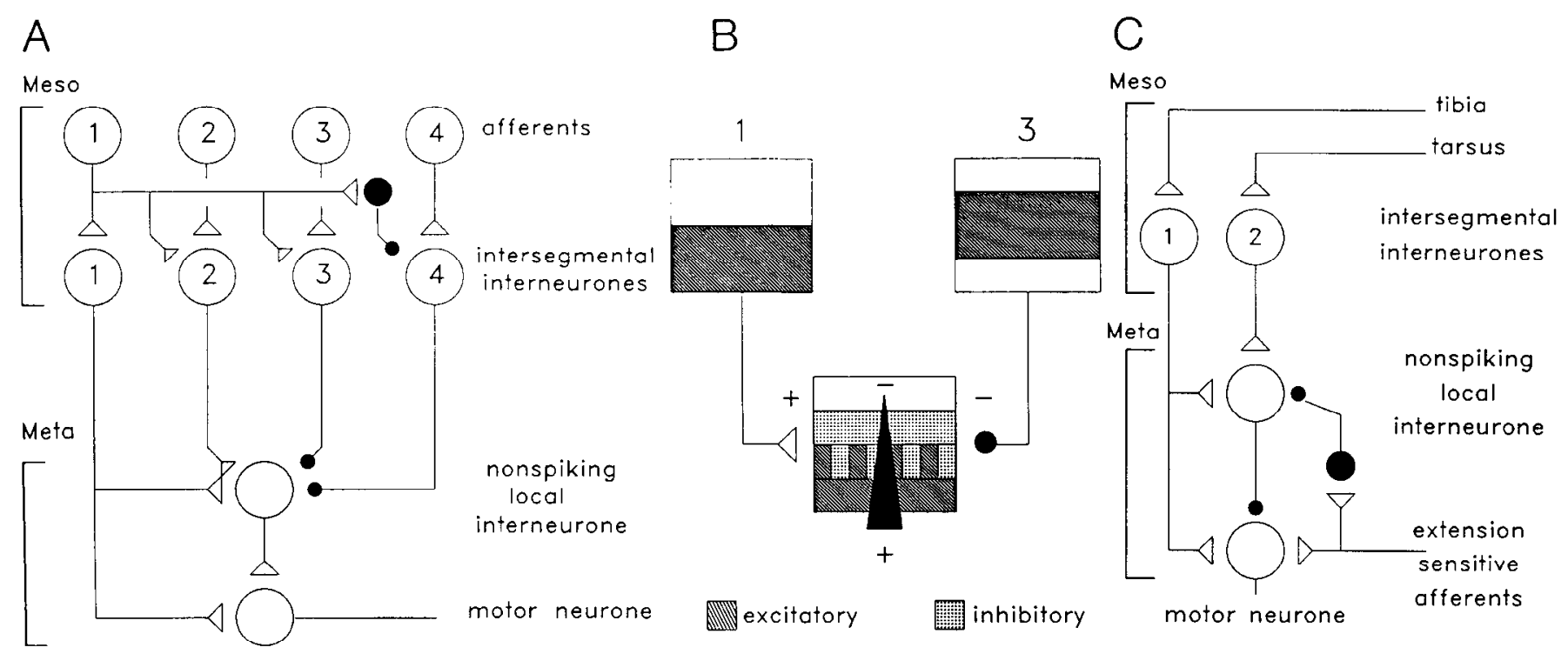

Figure 9. Flow diagrams of intersegmental pathways. A, Patterns of connections found between mesothoracic afferents and intersegmental interncurons and metathoracic nonspiking interneurons and motor neurons (triangles, excitatory; circles, inhibitory connections). All types of connection have been found in separate experiments and collated in this summary diagram. The large filled circle represents a spiking local interneuron. (See text for further details.) $B$, Two intersegmental interneurons ( 1 and 3 in $A$ ), with overlapping excitatory receptive fields but opposite effects, converge on a nonspiking interneuron. This could result in a gradient (filled triangle) of inhibition and excitation. $C$, Metathoracic nonspiking interneurons are a point of convergence of local and intersegmental inputs. The diagram represents the simplest pathways explaining the experimental findings in Figure 8. The large filled circle represents a spiking local interneuron. Local inputs to the nonspiking interneuron can affect the flow of intersegmental information to the metathoracic motor neuron.

the definition of their mesothoracic receptive fields (Laurent, 1987b). Second, as revealed here, it may assist other intersegmental interneurons with outputs of the opposite polarity that converge onto the same metathoracic target. This might explain why certain mesothoracic intersegmental interneurons have inhibitory regions in their receptive field not contiguous to the excitatory ones and whose function remained unclear until their output connections were discovered. It will thus be important to study the maturation of receptive field properties of intersegmental interneurons during postembryonic development. The results shown in Figure 3 suggest that the establishment of the local inhibitory connectivity between spiking local interneurons and intersegmental interneurons might depend on the output properties of the intersegmental interneurons. It is interesting to compare this experimental finding with a recent modeling experiment. In a 3-layered neural network model designed to determine shape from shading (Lehky and Sejnowski, 1988), hidden units develop precise receptive fields easily predictable from their patterns of inputs, but not easily interpreted in terms of their function. Lehky and Sejnowski (1988) concluded that, in this example, "understanding" the receptive field of a unit appears to depend as much on knowing its output connections ("projective field") as its pattern of inputs.

\section{Definition of a receptive field}

A metathoracic nonspiking interneuron can have a complex mesothoracic receptive field that results from the convergence of inputs from several mesothoracic intersegmental interneurons. The complexity of the pathways and the number of interneurons acting in parallel, however, make it difficult to determine a receptive field accurately. Transmission in each of the parallel pathways will not necessarily be reliable because each neuron receives inputs from many sources that can change its excitability. For example, the excitatory region of the nonspiking interneuron in Figure $9 A$ that results from the disinhibition of the mesothoracic interneuron 4 will only be apparent if the latter is already exerting its inhibitory effect, when receptors represented by sensory neuron 4 are activated. Similarly, the apparent extent of the excitatory receptive field will depend on whether interneuron 1 alone is activated or whether interneuron 2 also is recruited. Care must, therefore, be exercised in defining the receptive field of an interneuron that is more than 2 synapses away from an array of sensory neurons.

\section{Nonspiking interneurons as summing points of local and intersegmental input}

The mesothoracic receptive fields of metathoracic nonspiking local interneurons result from convergent input from several intersegmental interneurons, which themselves receive overlapping inputs. The responses of a metathoracic nonspiking local interneuron to a precise mesothoracic stimulus will thus largely depend on the context in which they occur. The nonspiking local interneurons release transmitter in a graded fashion (Burrows and Siegler, 1978), so that the richness and variety of inputs from several intersegmental interneurons could be encoded precisely and delivered to the motor neurons. The nonspiking interneurons also are directly involved in local reflex pathways as intermediates betwecn sensory afferents from the hindleg and motor neurons (Burrows et al., 1988; Laurent and Burrows, $1988 \mathrm{~b}$ ). They are thus a point for convergence and summation of intersegmental and local sensory signals. Figure $9 \mathrm{C}$ summarizes the simplest pathways needed to explain the interactions between meso- and metathoracic stimuli described in Figure 8. The effect of mesothoracic tibial afferents on the metathoracic motor neuron will be enhanced if inputs from the metathoracic extension-sensitive afferents occur simultaneously 
and reduced if mesothoracic tarsal inputs occur. Similarly, the cffect of metathoracic extension-sensitive afferents will depend on the occurrence of mesothoracic inputs from the tibia or tarsus. The operation of local reflexes must, therefore, be considered in the wider context of sensory inputs provided to the other legs.

The predictions made above assume that the effects of a nonspiking interneuron on a postsynaptic motor neuron are simply equal to the sum of all the inputs it receives. Nonspiking local interneurons in arthropods, however, have widely distributed neuropilar branches (Siegler and Burrows, 1979; Watkins et al. 1985), with closely apposed input and output synapses (Kondoh and Hisada, 1986; Watson and Burrows, 1988) that could form electronically independent circuits within a single neuron. If such effects do occur, many other factors must be considered than the nature and polarity of intersegmental inputs in modulating local reflexes. These possibilities are considered in the following paper (Laurent and Burrows, 1989).

\section{References}

Bässler, U. (1983) Neural Basis of Elementary Behaviour in Stick Insects. Studies of Brain Function 10, Springer-Verlag, Berlin.

Burrows, M. (1979) Graded synaptic transmission between local premotor interneurons of the locust. J. Neurophysiol. 42: 1108-1123.

Burrows, M. (1980) The control of sets of motoneurones by local interneurones in the locust. J. Physiol. (Lond.) 298: 213-233.

Burrows, M. (1987a) Parallel processing of proprioceptive signals by spiking local interneurons and motorneurons in the locust. J. Neurosci. 7: 1064-1080.

Burrows, M. (1987b) Inhibitory interactions between spiking and nonspiking local interneurons in the locust. J. Neurosci. 7: 3282-3292.

Burrows, M., and M. V.S. Siegler (1978) Graded synaptic transmission between local interneurones and motoneurones in the metathoracic ganglion of the locust. J. Physiol. (Lond.) 285: 231-255.

Burrows, M., and M. V. S. Siegler (1982) Spiking local interneurons mediate local reflexes. Science (NY). 217: 650-652.

Burrows, M., and M. V. S. Siegler (1984) The morphological diversity and receptive fields of spiking local interneurons in the locust metathoracic ganglion. J. Comp. Neurol. 224: 483-508.

Burrows, M., G. J. Laurent, and L. H. Field (1988) Proprioceptive inputs to nonspiking local interneurons contribute to local reflexes of a locust hindleg. J. Neurosci. 8: 3085-3093.

Capaday, C., and R. B. Stein (1986) Amplitude modulation of the soleus H-reflex in the human during walking and standing. $J$. Neurosci. 6: 1308-1313.

DiCaprio, R. A., and F. Clarac (1980) Reversal of a walking leg reflex elicited by a muscle receptor. J. Exp. Biol. 90: 197-263.

Diet7, V., J. Quintern, G. Boos, and W. Berger (1986) Obstruction of the swing phase during gait: Phase-dependent bilateral leg muscle coordination. Brain Res. 384: 166-169.

Feldman, A. G., and G. N. Orlovsky (1975) Activity of interneurons mediating reciprocal inhibition during locomotion. Brain Res. 84: 181-194.

Forssberg, H. (1979) Stumbling corrective reaction: A phase-dependent compensatory reaction during locomotion. J. Neurophysiol. 42: 936-953.

Graham, D. (1985) Pattern and control of walking in insects. Adv. Insect. Physiol. 18: 31-140.

Grillner, S. (1981) Control of locomotion in bipeds, tetrapods, and fish. In Handbook of Physiology, The Nervous System, Motor Control, V. B. Brooks, ed., Sect. 1, vol. 2, pp. 1 179-1236, American Physiology Society, Bethesda, MD.
Hasan, Z., and D. G. Stuart (1988) Animal solutions to problems of movement control: The role of proprioceptors. Annu. Rev. Neurosci. 11: 199-223.

Jankowska, E., and W. J. Roberts (1972) Synaptic action of single interneurons mediating reciprocal $\bar{I}_{a}$ inhibition of motoneurones. J. Physiol. 222:623-642.

Kondoh, Y., and M. Hisada (1986) Distribution and ultrastructure of synapses on a premotor local nonspiking interneuron of the crayfish. J. Comp. Neurol. 254: 259-270.

Laurent, G. (1986) Thoracic intersegmental interneurons in the locust with mechanoreceptive inputs from a leg. J. Comp. Physiol. 159: $171-186$.

Laurent, G. (1987a) The morphology of a population of thoracic intersegmental interneurons in the locust. J. Comp. Neurol. 256:412429.

Laurent, G. (1987b) The role of spiking local interneurons in shaping the receptive fields of intersegmental interneurons in the locust. $J$. Neurosci. 7: 2977-2989.

Laurent, G. (1988) Local circuits underlying excitation and inhibition of intersegmental interneurons in the locust. J. Comp. Physiol. 162. $145-157$

Laurent, G., and M. Burrows (1988a) A population of ascending intersegmental interneurons in the locust with mechanosensory inputs from a hind leg. J. Comp. Neurol. 275: 1-12.

Laurent, G. J., and M. Burrows (1988b) Direct excitation of nonspiking local interneurons by exteroceptors underlies tactile reflexes in the locust. J. Comp. Physiol. 162: 563-572.

Laurent, G. J., and M. Burrows (1989) Intersegmental interneurons can control the gain of reflexes in adjacent segments of the locust by their action on nonspiking local interneurons. J. Neurosci. 9: 30303039.

Laurent, G. J., and R. Hustert (1988) Motorneuronal receptive fields delimit patterns of activity during locomotion of the locust. J. Neurosci. 8: 4349-4366.

Lehky, S. R., and T. J. Sejnowski (1988) Network model of shapeform-shading: Neural function arises from both receptive and projective fields. Nature 333: 452-454.

Pearson, K. G., and R. M. Robertson (1987) Structure predicts synaptic function of two classes of interneurons in the thoracic ganglia of Locusta migratoria. Cell Tissue Res. 250: 105-114.

Siegler, M. V. S., and M. Burrows (1979) The morphology of local nonspiking interneurons in the metathoracic ganglion of the locust. J. Comp. Neurol. 183: 121-148.

Siegler, M. V. S., and M. Burrows (1983) Spiking local interneurons as primary integrators of mechanosensory information in the locust. J. Neurophysiol. 50: 1281-1295.

Siegler, M. V. S., and M. Burrows (1986) Receptive fields of motorneurons underlying local tactile reflexes in the locust. J. Neurosci. 6 : 507-513.

Sillar, K. T., and A. Roberts (1988) A neuronal mechanism for sensory gating during locomotion in a vertebrate. Nature 331: 262-265.

Sillar, K. T., and P. Skorupski (1986) Central input to primary afferent neurones in crayfish, Pacifastacus leniusculus, is correlated with rhythmic output of thoracic ganglia. J. Neurophysiol. 55: 678-688.

Watkins, B. L., M. Burrows, and M. V. S. Siegler (1985) The structure of locust nonspiking interneurons in relation to the anatomy of their segmental ganglion. J. Comp. Neurol. 240: 233-255.

Watson, A. H. D. (1986) The distribution of GABA-like immunoreactivity in the thoracic nervous system of the locust Schistocerca gregaria. Cell Tissue Res. 246: 331-341.

Watson, A. H. D., and M. Burrows (1988) The distribution and morphology of synapses on nonspiking local interneurons in the thoracic nervous system of the locust. J. Comp. Neurol. 272: 605-616. 